\title{
PROFILE OF LOSS CONE ON PLASMA SURFACE OF THE 14.4 GHz ECR ION SOURCE IN KOLKATA
}

\author{
M. H. Rashid* and R. K. Bhandari \\ Variable Energy Cyclotron Centre (DAE), Sector-1, Block-AF, Bidhan Nagar, \\ Kolkata-700 064, INDIA.
}

\begin{abstract}
Recently a $14.4 \mathrm{GHz}$ ECRIS [1] was designed for the superconducting cyclotron under construction. It has the conventional min-B (Ioffe) configuration for reflecting the escaping electrons. The electrons gyrating around a line of force falling in a loss cone (LC) will not be reflected by the mirror field. So, it is important to have the knowledge of LC in the inner space of the plasma chamber which is dependent on the position of creation and subsequent motion of electrons and value of the mirror ratio (MR). The equation for evaluating the $\mathrm{LC}$ is formulated and used for LC calculation from the MR at a point. The MR thereby giving the LC profile around the resonance surface is evaluated.
\end{abstract}

\section{LOSS CONE}

An LC signifies the loss of electrons falling into it unless it is knocked out of it by some physical processes. So, it is important to evaluate it quantitatively which gives qualitative idea of the loss of electrons all around the plasma chamber. It gives qualitative idea because its motion is vigorously affected by many other physical phenomena like collision, diffusion etc. So for the first time an attempt has been made to calculate it on a surface just enclosing the resonance zone and evaluate the reflection co-efficient of electrons at a point of its creation on the surface and subsequent motion.

\subsection{Formulation}

We assume a line of force QSOF in the plasma chamber which encounters the cylindrical wall or end of the chamber in both the forward and backward direction of the line (Fig. 1a). Let an electron gyrates around the line of force at $\mathrm{O}$ with total momentum $P=|\mathbf{P}|$ having components parallel and perpendicular to the line of force $P_{\| \mid}$and $P_{\perp}$ respectively. A remarkable point is that the electrons may have $P_{\|}$, parallel or antiparallel i.e. it may move forward (in positive direction) or backward (in negative direction) w. r. t. the line of force. The magnetic field at the two points where the lines of force meet the wall may be different and represented by $\mathrm{B}_{\mathrm{s}+}$ and $\mathrm{B}_{\mathrm{s}-}$ in parallel and antiparallel direction respectively. The local magnetic field at $\mathrm{O}$ is $\mathrm{B}_{\mathrm{o}}$ and $\mathrm{B}_{\min }$ is the minimum field at certain point on the line of force. The equations (1a) and (1b) give the local MRs in forward and backward directions respectively at the point $\mathrm{O}$ whereas equation (1c) give the $\mathrm{R}$ in common use so far defined traditionally. The function $\max \left(\mathrm{B}_{\mathrm{s}^{+}}, \mathrm{B}_{\mathrm{s}-}\right)$ in equation (1c) selects maximum of the two values.

$R_{+}=B_{s+} / B_{o}$

$R_{-}=B_{s-} / B_{o}$

$R_{m}=\max \left(B_{s^{+}}, B_{s_{-}}\right) / B_{\text {min }}$

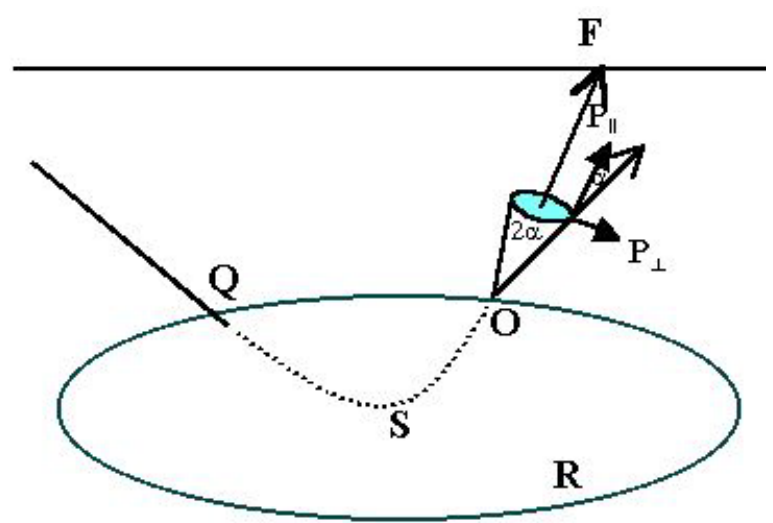

Figure 1(a): An electron is created at $\mathrm{O}$ on the line of force and moves around $\mathrm{OF}$ at an angle $\alpha$.

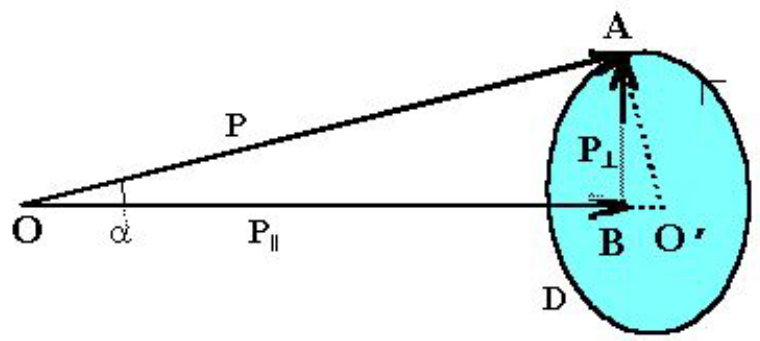

Figure 1(b) : The shaded elliptical portion is a part of the assumed spherical surface with radius $P$ (magnitude of momentum). The line of force passes along the line OO' (through the centre of the shaded dish).

The MR, $R_{m}$ gives a qualitative feeling of the tightness of the plasma confinement and the efficiency of the multiply stripped ion production in the plasma. But as far as the production of electron at certain position and

*E-mail: haroon@veccal.ernet.in 
subsequent motion of the electron is concerned the concept of local MRs, $R_{+}$and $R_{-}$are very important for evaluation and estimation of electron reflection or loss. The actual angle of the cone of spiralling electron around the line of force and apex angle of the LC is given by equation $2 \mathrm{a}$ and $2 \mathrm{~b}$ respectively.

The solid angle of the LC is deduced from figure (1b). Let an electron is at $\mathrm{O}$ and taking the magnitude of the momentum of electron as radius we imagine a sphere around $\mathrm{O}$. The circular shaded portion enclosed by the gyrating electron is a part of the sphere. The area (S) of the shaded dish and the solid angle of the electron is given by equations 3 and 4a respectively. Using the apex angle obtained by the adiabatic invariance of the magnetic moment of electron we obtain the solid angle of the LC given by equation $4 \mathrm{~b}$.

$$
\begin{aligned}
& \alpha=\sin ^{-1}\left(P_{\perp} / P\right) \\
& \alpha_{\text {apex }, i}=\sin ^{-1}\left(1 / \sqrt{R_{i}}\right) \\
& S=2 \pi P\left(P-P_{\|}\right)=2 \pi P^{2}(1-\cos \alpha) \\
& d \Omega=2 \pi(1-\cos \alpha) \\
& d \Omega_{i}=2 \pi\left(1-\cos \left(\alpha_{\text {apex }, i}\right)\right)
\end{aligned}
$$

where, $\mathrm{R}_{\mathrm{i}} \in\left\{\mathrm{R}_{+}, \mathrm{R}_{-}, \mathrm{R}_{\mathrm{m}}\right\}$. That is, the subscript $i=+,-$ or $\mathrm{m}$.

If $\quad \alpha<\alpha_{a p e x, i}$ then the electron falls inside the LC and there is sound probability of being lost unless it switches over to another position and starts moving from the point out of the LC afresh.

\subsection{Evaluation}

The MRs, $R_{+}, R_{-}$or $R_{m}$ at any point in the chamber are given by TrapCAD $[2,3]$ on the line of force passing through the point after properly feeding the coil and multipole magnetic field data. If the local field at the point, we assume the field $\left(B_{o}\right)$ at point $O$, is known then all the fields $\mathrm{B}_{\mathrm{s+}}, \mathrm{B}_{\mathrm{s}-}$ and $\mathrm{B}_{\min }$ can be calculated readily using the obtained values from above formulae of MRs.

The apex angle $\alpha_{\text {apex }, i}$ and solid angle $\mathrm{d} \Omega_{i}$ of the LC were evaluated at the vicinity of the resonance surface (Fig. 2) in the chamber corresponding to the $R F$ frequency $14.4 \mathrm{GHz}$. The resonance surface is positioned from $-6.0 \mathrm{~cm}$ to $6.0 \mathrm{~cm}$ w. r. t. the centre. The values of $\mathrm{d} \Omega_{i}$ were evaluated in the parallel and anti-parallel directions of the lines of force along the whole plasma length and from $30^{\circ}$ to $90^{\circ}$ azimuth due to field symmetry (Fig. 3 and 4). The sum of the two gives the total solid angle ( $\mathrm{d} \Omega_{t}=\mathrm{d} \Omega_{+}+\mathrm{d} \Omega_{\text {. }}$ ) at a point. The LC was evaluated using the traditional $M R, R_{m}$ also (Fig. 5) inside the plasma chamber.

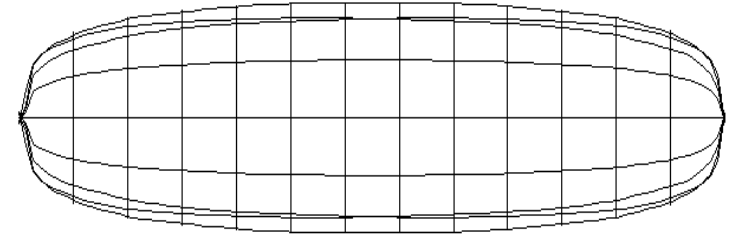

Figure $2:$ The resonance field surface at $5.143 \mathrm{kG}$ have $3.5 \mathrm{~cm}$ diameter at the mid-length. The centre meridian is at $0^{0}$ along the length and others at $30^{\circ}$ gap.

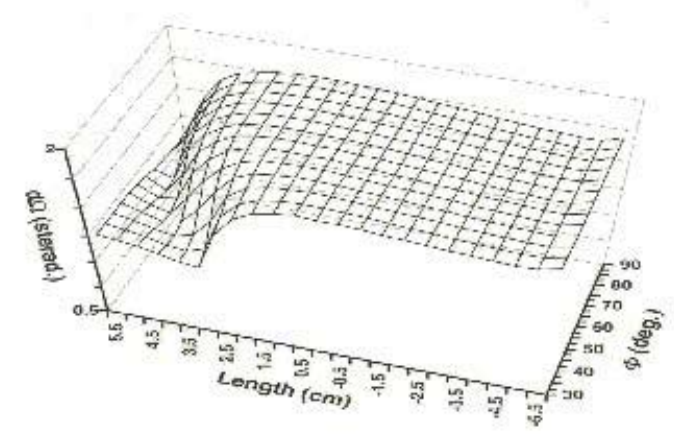

Figure 3 : The LC plot for parallel motion of electrons w.r.t. the lines of force (i.e. using $M R, R_{+}$).

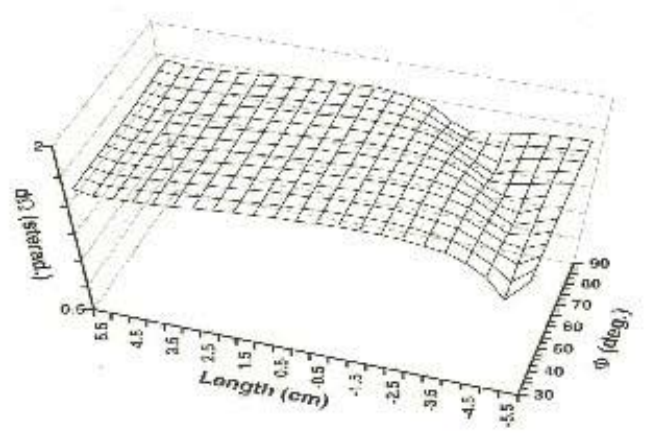

Figure 4 : The LC plot for anti-parallel motion of electrons w.r.t. the lines of force (i.e. using MR, R.).

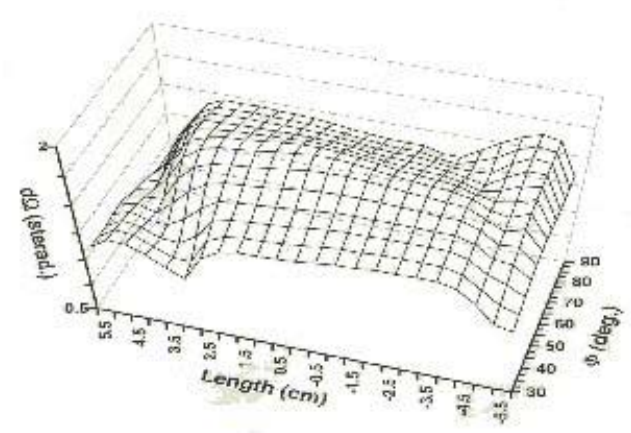

Figure 5 : The LC plot for motion of electrons using traditional $\mathrm{MR}, \mathrm{R}_{\mathrm{m}}$. 


\section{DISCUSSION}

The field configuration is 3 -fold symmetric along the azimuth because of sextupolar radial field. The loss holes are formed at $90^{\circ}, 210^{\circ}$ and $330^{\circ}$ azimuth at the extraction side for electrons moving towards the negative direction of the field i. e. towards the extraction. The azimuthal position of the loss hole is rotated by $60^{\circ}$ at the injection side for the electrons moving towards the positive direction of the field i. e. towards the injection due to the presence of reverse radial field component of the axial field at the extraction side. But these loss hole positions are far away from the resonance field surface at the ends and one should not be alarmed with it.

It is seen from the figures 3 and 5 that the $\mathrm{LC}$ at the mid-length of the plasma surface are more or less constant while using the lacal MRs and the electrons have least LC while moving towards the mirror peak after scattering or creation. It is apparent from the calculation that the electrons which start moving from inside the resonance surface has smaller LC than the electrons starting motion from outside the surface. One can easily find the position of the magnetic hole where maximum loss of the electrons take place (Fig. 5).

The loss of the electrons takes place inherently since $\mathrm{d} \Omega_{t} \neq 0$ at any point. But how much loss at the point happens? First, we considers the point of creation of electrons $(\mathrm{O})$ on the line of force in the plane, 2D surface, containing the line of force. The electrons move isotropically about the point then the reflection coefficient $\left(R_{c o-e f f ;} i\right)$ is given by the equation 5a [4].

$$
R_{c o-e f f, i}=\cos ^{2}\left(\alpha_{\text {apex.i }}\right)=1-R_{i}
$$

But this is not the reality, one has to take into account the three dimensional situation at the point because a particle may move in any direction after creation there. A quantitative measure is given by $\mathrm{d} \Omega_{i} / 2 \pi$ at a point in the direction of the drift of the gyrating electron in a special case in which we assume for an instance that there is no effect on motion of electrons due to plasma, they are absolutely guided by the electromagnetic field present in the chamber, production and scattering of electrons takes place isotropically at a point and it is highly random. Now the reflection coefficient, $R_{c o \text {-eff. } i}$, of the electron at a point is given by equation $5 \mathrm{~b}$.

$$
R_{\text {co-eff, } i}=1-\left(\mathrm{d} \Omega_{i} / 2 \pi\right)=\cos \left(\alpha_{\text {apex.i }}\right)
$$

As soon as the plasma comes into picture the LC is affected by it tremendously through many physical processes like stepwise ionisation of atoms and binary or collective, elastic or inelastic scattering and multiplication of electrons in the chamber.

According to the expression of LC, MR solely decides value of the $\mathrm{LC}$. The source should have sufficient MR to ensure the minimum LC angle. The field configuration in the chamber should confirm it such that the magnetic field surface for electron cyclotron resonance is sufficiently far off the inner surface of the chamber for the accelerated electrons to get sufficient length of flight for incessant change of position to take place by some physical means. The local MR on certain point on that surface should be sufficiently high to get the lowest possible local LC to get the electrons reflected for avoiding loss on the chamber surface on hitting it.

The $R_{\mathrm{m}} \cong 3.0$ on the resonance surface. The electrons on ECR region get transverse energy over a small axial distance while in interaction with micro-wave, so the $P_{\perp}$ component of the momentum increases. The electrons conserve the momentum acquired while in resonance for a few microseconds and subsequently it remains perpendicular to the magnetic field line. A strong anisotropy of momentum components, $P_{\perp} / P_{\|}>1$, will occur [5]. Downstream from the interaction region the energised electrons are magnetically confined and can ionise the background gas. Evaluation of the LCs taking into account the major cause of effects in the whole space of the plasma chamber gives very significant information about the plasma confinement and loss of electrons. Further, it can give a figure of merit or qualitative value of efficiency of an ECRIS.

\section{REFERENCES}

[1] M. H. Rashid et. al., Proceedings of this conference.

[2] J. Vamosi and S. Biri, Nucl. Instr. and Meth., B94, (1994), 297.

[3] S. Biri and J. Vamosi, Proc. of the EPAC 94, Vol.-2, (1994), 1432.

[4] S. R. Seshadri, Fundamentals of Plasma Physics, American Elsivier Publishing Co. Inc., New York, (1973), 184.

[5] R. Geller et.al., Proc. of the $12^{\text {th }}$. Int. Workshop on ECR Ion Source, Riken, (1995), 4. 